\title{
3. \\ Note sur le §. 6 du mémoire No.9. inséré dans le tome 43 de ce journal.
}

(Par Mr. Steichen, professeur à l'école milit. à Bruxelles.)

Dans la rémarque II du $\mathfrak{S}$. cité jai admis que les formules qui donnent la direction de l'axe d'ébranlement du solide, conservent la même forme simple qu'elles ont pour les axes permanents du point fixe $(O)$, alors même qu'on passe à d'autres axes rectangles quelconques $O x^{\prime}, O y^{\prime}, O z^{\prime}$.

Cette assertion n'est pas exacte. Néanmoins il existe outre le centre de percussion donné par la théorie ordinaire, une autre solution de la question que le \$. 6 ne fournit que par le moyen de certaines modifications que je vais indiquer.

$C_{1}^{\prime}, B_{1}^{\prime}, A_{1}^{\prime}$, étant toujours les angles de l'axe d'ébranlemeut $O K$ avec des axes rectangles arbitraires $\left(O x^{\prime}, O y^{\prime}, O z^{\prime}\right)$, et $d \xi$ la rotation résultante; $d \psi^{\prime}, d \omega^{\prime}$, $d \varphi^{\prime}$ ses composantes autour de ces axes. Posons, pour abréger l'écriture:

$$
\frac{2 d \xi}{d t^{2}}=\xi^{\prime}, \frac{2 d \psi^{\prime}}{d t^{2}}=\psi^{\prime}, \frac{2 d \omega^{\prime}}{d t^{2}}=\omega^{\prime}, \frac{2 d \varphi^{\prime}}{d t^{2}}=\varphi^{\prime},
$$

et nous aurons (page 187) pour les composantes rectangles au point $\left(x^{\prime}, y^{\prime}, z^{\prime}\right)$ de la molécule $d m$, provenant de sa réaction d'inertie, les valeurs suivantes:

$$
\begin{aligned}
& \text { d. } \Pi_{x^{\prime}}=\omega^{\prime} \cdot z^{\prime} d m-\varphi^{\prime} \cdot y^{\prime} d m, \\
& \text { d. } \Pi_{y^{\prime}}=\varphi^{\prime} \cdot x^{\prime} d m-\psi^{\prime} \cdot z^{\prime} d m, \\
& d . \Pi_{z^{\prime}}=\psi^{\prime} \cdot y^{\prime} d m-\omega^{\prime} \cdot x^{\prime} d m .
\end{aligned}
$$

De là on conclut aisément que le moment $d \Pi_{x^{\prime}} y^{\prime}-d \Pi_{r^{\prime}} x^{\prime}$ de cette force élementaire a la valeur autour de l'axe $O z^{\prime}$ :

$$
\omega^{\prime} \cdot y^{\prime} z^{\prime} d m-\varphi^{\prime}\left(y^{\prime 2}+x^{\prime 2}\right) d m+\psi^{\prime} x^{\prime} z^{\prime} d m ;
$$

ce qui donne pour la somme des moments des réactions d'inertie autour de l'axe $\boldsymbol{O}^{\prime}$ la valeur suivante:

$$
\mathfrak{N}_{z^{\prime}}=\psi^{\prime} \int x^{\prime} z^{\prime} d m+\omega^{\prime} \int y^{\prime} z^{\prime} d m-\varphi^{\prime} \int\left(y^{2}+x^{2}\right) d m .
$$


Si donc on désigne par $A^{\prime}, B^{\prime}, C^{\prime}$ les moments d'inertie du solide, relatifs à $O x^{\prime}, O y^{\prime}, O z^{\prime}$, et que l'on fasse en outre

$$
\int x^{\prime} y^{\prime} d m=g, \int y^{\prime} z^{\prime} d m=h, \int z^{\prime} x^{\prime} d m=k,
$$

on obtiendra pour les valeurs des moments des réactions autour des axes $\boldsymbol{O}^{\prime}$, $O x^{\prime}, O y^{\prime}$, les formules:

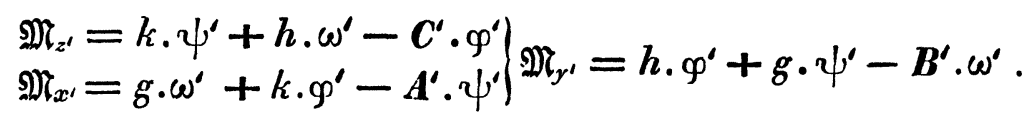

Or puisqu'il y a équilibre entre l'action et les réactions d'inertie tangentielles (car les réactions d'inertie normales n'existent pas encore pendant l'instant du mouvement naissant, ou sont du moins insensibles): il faut que la somme des moments de ces forces autour de chaque axe coordonné en $(O)$, soit'nulle. Cela donne les équations nécessaires et suffisantes:

$$
\mathfrak{M}_{x^{\prime}}+N^{\prime}=0, \mathfrak{M}_{y^{\prime}}+M^{\prime}=0, \mathfrak{M}_{z^{\prime}}+L^{\prime}=0,
$$

ou bien

(1.) $A^{\prime} \psi^{\prime}-g \omega^{\prime}-k . \varphi^{\prime}=N^{\prime}, B^{\prime} \cdot \omega^{\prime}-h . \varphi^{\prime}-g \psi^{\prime}=M^{\prime}, C^{\prime} . \varphi^{\prime}-k \psi^{\prime}-h . \omega^{\prime}=L^{\prime}$, et l'intégration donne immédiatement pour les composantes totales des forces d'inertie elles - mêmes :

(2.) $\Pi_{x^{\prime}}=\omega^{\prime} \cdot \mu . z_{1}^{\prime}-\varphi^{\prime} \cdot \mu \cdot y_{1}^{\prime} ; \Pi_{y}=\varphi^{\prime} \cdot \mu \cdot x_{1}^{\prime}-\psi^{\prime} \cdot \mu . z_{1}^{\prime} ; \Pi_{z^{\prime}}=\psi^{\prime} \mu y_{1}^{\prime}-\omega^{\prime} \mu x_{1}^{\prime}$. $\mu$ exprime la masse du solide, et $x_{1}^{\prime} y_{1}^{\prime} z_{1}^{\prime}$ sont les coordonnées du centre d'inertie à l'instant de l'ébranlement.

On voit sur le champ par les formules (I) que l'on peut seulement avoir:

$$
\frac{2 d \psi}{d t^{2}}=\frac{N}{A}, \frac{2 d \omega}{d t^{2}}=\frac{M}{B}, \frac{2 d \varphi}{d t^{2}}=\stackrel{L}{C},
$$

quand les axes coordonnés coïncident avec les axes permanents en $(O)$ pour lesquels les lettres non-accentuèes ont la même signification que les accentuées, par rapport aux trois axes $\left(O x^{\prime}, O y^{\prime}, O z^{\prime}\right)$, et dès lors seulement aussi les formules (2) donneraient:

$$
\Pi_{x}=\frac{M}{B} \cdot \mu z_{1}-\frac{L}{C} \cdot \mu y_{1} \quad, \quad \Pi_{y}=\ldots . . \quad, \quad \Pi_{z}=\ldots .
$$

Si donc il y a en dehors de la solution de la théorie ordinaire, une autre solution encore, il faut que l'analyse précédente, expimant le tout sous une forme générale, la fournisse. Or en nommant $\boldsymbol{P}$ la force agissante et $(\alpha, \beta, \gamma)$ sa direction, il faut d'abord que l'on ait:

$$
\Pi_{x^{\prime}}+\boldsymbol{P}_{\alpha}=\mathbf{0}, \Pi_{r}+\boldsymbol{P} \cdot \beta=\mathbf{0}, \quad \Pi_{z^{\prime}}+\boldsymbol{P} \cdot \gamma=\mathbf{0}
$$

afin d'avoir une percussion nulle sur le point fixe $(O)$; car l'axe d'ébranlement ne saurait souffrir évidemment d'autre effort qu'en ce point. Mais nous voyons 
qu'en dirigeant les axes $\left(O x^{\prime}, O y^{\prime}, O z^{\prime}\right)$ restés jusqu'ici arbitraires, le premier suivant l'axe $O I$, qui joint le point fixe au centre $I$, et les deux autre d'abord dans le plan normal, on obtient $y_{1}^{\prime}=0, z_{1}^{\prime}=0$, partant $\Pi_{x^{\prime}}=0$ : et de là résulte $\boldsymbol{P} \alpha=0$, ou $\alpha=0$; ce qui prouve que la force $\boldsymbol{P}$ doit croiser la ligne $O I$ sous un angle droit. Si donc on choisit l'axe $O y^{\prime}$ parallèle à la ligne de $P$, on obtient en outre $\beta=1, \gamma=0$; partant:

$$
\boldsymbol{P}+\Pi_{y^{\prime}}=\mathbf{0}, \text { et } \Pi_{z^{\prime}}=\mathbf{0} \text {. }
$$

Substituant dans ces expressions les valeurs de $\Pi_{\gamma^{\prime}}, \Pi_{z^{\prime}}$ données par les formules (2), dans l'hypothèse de $y_{1}^{\prime}=0, z_{1}^{\prime}=0$, on en déduit:

$$
\boldsymbol{P}+\varphi^{\prime} \mu . x_{1}^{\prime}=0 \text {, et } \omega^{\prime}=0 \text {. }
$$

En outre la même disposition des axes coordonnés, donne, en nommant $(a, b, c)$ ou simplement $(a, c)$ les coordonnées du point d'àpplication de la force $\boldsymbol{P}$ : $\boldsymbol{L}^{\prime}=\boldsymbol{P}(a . b-\beta . a)=-\boldsymbol{P a} \quad, \quad N^{\prime}=\boldsymbol{P}(\beta c-\gamma b)=\boldsymbol{P} . c \quad, \quad \boldsymbol{M}^{\prime}=\boldsymbol{P}(\gamma a-\alpha c)=\mathbf{0}$; ainsi aux deux conditions (a) il faut joindre cette troisième:

$$
M^{\prime}=\mathbf{0} \text {. }
$$

Par cette dernière $(b)$ et la dernière (a) les équations (I) deviennent:

$$
\text { (c.) } A^{\prime} \psi^{\prime}-k \varphi^{\prime}=\text { P.c }, h \cdot \varphi^{\prime}+g \psi^{\prime}=0, C^{\prime} \cdot \varphi^{\prime}-k \cdot \psi^{\prime}=-P . a
$$

Les deux dernières donneront les valeurs

$$
\begin{gathered}
\varphi^{\prime}=-\frac{P a g}{C^{\prime} g+h k} \quad, \quad \psi^{\prime}=+\frac{P \cdot a \cdot h}{C^{\prime} g+h \cdot k} \quad, \quad \xi^{\prime}=\frac{P \cdot a \cdot V\left(g^{2}+h^{2}\right)}{C^{\prime} g+h \cdot k} \\
\cos C_{1}^{\prime}=\frac{\psi^{\prime}}{\xi^{\prime}}=\frac{h}{V\left(g^{2}+h^{2}\right)} \quad, \quad \cos A_{1}^{\prime}=-\frac{g}{V\left(g^{2}+h^{2}\right)}, \quad \cos B_{1}^{\prime}=\omega^{\prime}: \xi^{\prime}=0 .
\end{gathered}
$$

En substituant les valeurs de $\varphi^{\prime}, \psi^{\prime}$ dans la première $(C)$, on obtient entre $a$ et $c$ la condition:

$$
\left(C^{\prime} g+h . k\right) \cdot c-\left(A^{\prime} h+g k\right) a=0 .
$$

Enfin. portant dans la première (a) la valeur de $\varphi^{\prime}$, on en tire:

$$
\text { (e.) } \quad a=\frac{C^{\prime} \cdot g+h k}{\mu \cdot g \cdot O I}, \text { d'où } \quad c=\frac{A^{\prime} h+g k}{\mu \cdot g \cdot O I} \text {; }
$$

car l'abscisse $x_{1}^{\prime}$ est évidemment mesurée par la droite $O 1$. Ainsi donc, pour avoir un effort d'action et de réaction nul sur le point fixe donné, il faut faire agir la force suivant une direction qui croise l'axe $O I$ sous un angle droit; ce qui revient à la diriger normalement à un plan quelconque $\boldsymbol{P}$ mené suivant la droite $O I$. En nommant $O y^{\prime}$ la normale en $(O)$ à ce plan $P$, et $O z^{\prime}$ un axe situé daus $\boldsymbol{P}$ et rectangle avec $O I$, on évaluera les quantités $g=\int x^{\prime} y^{\prime} d m^{\prime} h=\ldots ., k=\ldots$ relatives à ces axes; on calculera ensuite $A^{\prime}, C^{\prime}$ par la règle connue, et par les formules (e) on obtient enfin les coordonncés $(a, c)$ du centre de percussion dans 
le plan $\boldsymbol{P}$, où il faut faire agir la force pour avoir une secousse absolument nulle sur l'axe et sur le point fixe donné. Ces conditions étant satisfaites, on voit que l'axe d'ébranlement est toujours situé dans le plan $\boldsymbol{P}$ auquel la force est perpendiculaire.

Enfin pour prouver par une simple vérification que la solution nouvelle diffère essentiellement de celle de la théorie ordinaire, quoique dans l'une comme dans l'autre la force doive agir suivant une direction perpendiculaire au plan central mené par l'axe de rotation, il suffit de considérer que dans le cas actuel l'axe $\boldsymbol{O H}$ du moment principal de la force fait avec les axes coordonnés $O x^{\prime}, \boldsymbol{O}^{\prime}$ des angles de cosinus $c: V\left(a^{2}+c^{2}\right)$, et $-a: V\left(a^{2}+c^{2}\right)$, tandis que l'axe $O K$ fait avec ces axes des cosinus d'angle $h: V\left(g^{2}+h^{2}\right),-g: V\left(g^{2}+h^{2}\right)$, de sorte que les deux droites $\mathrm{OH}, O K$ n'ont en général que le seul point $(O)$ de commun; mais dans la solution ordinaire elle coïncident ensemble, et forment un axe permanent du solide au point fixe.

Si l'on resout les équations (1) par rapport aux quantités $\psi^{\prime}, \omega^{\circ}, \varphi^{\prime}$, on trouve:

$$
\psi^{\prime}=\frac{n\left(B^{\prime} C^{\prime}-h^{2}\right)+m\left(C^{\prime} g+h k\right)+l\left(B^{\prime} k+g h\right)}{A^{\prime} B^{\prime} C^{\prime}-A^{\prime} h^{2}-B^{\prime} k^{2}-C^{\prime} g^{2}-2 g h k} \quad, \quad \omega^{\prime}=\ldots . . \quad, \quad \varphi^{\prime}=\ldots . .
$$

Mais on peut aussi d'un autre coté obtenir ces mémes quantités à l'aide de celles $\psi=\frac{N}{A}, \omega=\frac{M}{B}, \varphi=\frac{L}{C}$, et du principe de la composition des rotations rectangulaires. En égalant ainsi ces doubles valeurs de chaque quantité $\psi^{\prime}, \omega^{\prime}, \varphi^{\prime}$, on obtiendrait trois équations de condition générales entre $A, B, C, A^{\prime}, B^{\prime}, C^{\prime}$, $g, h, k, N, M, L$, et neuf coëfficients de direction, lesquelles devraient subsister pour des valeurs quelconques des moments $N, M, L$; et par là on pourrait être ramené à la théorie des axes permanents de rotation des solides.

Bruxelles ce 27 avril 1852. 